\title{
Quantum non-demolition measurement in the interferometer
}

\author{
D.L. Khokhlov ${ }^{{ }^{*}}$ \\ ${ }^{1}$ Sumy State University (Retired), Ukraine \\ *Corresponding author E-mail:dlkhokhl@rambler.ru
}

\begin{abstract}
The schemes of the arrangements based on the Mach-Zehnder interferometer and the interferometer with two polarizing beam splitters are considered. The interferometers in both the schemes are equipped with some devices, creating a Kerr medium, to perform the quantum non-demolition measurement of a single photon. Such a device gives which way information of the photon while preserving the work of the interferometer.
\end{abstract}

Keywords: cavity quantum electrodynamics; interferometer; photon; quantum non-demolition measurement; which way information

\section{Introduction}

The outcomes of the interferometer and which way detector are orthogonal each other. It is thereby impossible to have both interference and which way information for a single particle in the same set up. This follows from the Bohr's complementarity principle [1]. The interplay of the interference and which way information is defined by the relationship of the complementarity parameters, visibility and distinguishability [2]. The problem can be represented in terms of wave-particle duality. To this end, an interpretation of wave-particle duality was suggested in [3, 4].

The Bohr's complementarity principle is about the projective measurement. Instead, one can apply other types of measurement, allowing detection of interference and which way information in the different configurations. The concept of weak measurement $[5,6]$ makes it possible to have both interference and which way information in the different configurations, in accordance with the Bohr complementarity. Several techniques of the weak measurement have been implemented with the flux of photons, e.g. [7, 8].

The scheme of the arrangement to perform the weak measurement of a single photon was suggested in [9]. The arrangement includes the modified Mach-Zehnder interferometer and which way detector. The system of the mirrors deflects the components of the photon, traveling two legs of the interferometer. Within the interferometer, the deflection is rather small thus not destroying interference. After the interferometer, the trajectories of the components of the photon diverge and may be distinguished at the which way detector. Following [9], the scheme of the modified interferometer with two polarizing beam splitters was considered [10]. One can use the arrangement [10] for attack on the protocol BB84 [11, 12].

The concept of quantum non-demolition (QND) measurement was designed to obtain information about the state, without destroying it, e.g. [13] and references therein. As a consequence, QND measurements yield identical results when repeated. In optical QND measurements, a signal beam is coupled to a meter in a Kerr medium, making a phase shift on the meter, depending on the intensity of the signal beam. A Kerr medium, working at the single photon level, can be realized in cavity quantum electrodynamics [14]. The QND detection of a single photon was demonstrated in the optical domain [15], and in the microwave domain [16, 17]. The detection technique is based on the strong-coupling regime of cavity quantum electrodynamics. A single photon is reflected off a cavity that leads to a phase flip of the atom in the cavity. Subsequent readout of the atomic phase allows to nondestructively detect the photon. Also, other techniques were realized to create a Kerr medium, dealing with single photons, e.g. [18, 19, 20].

The QND measurement of a single photon may be used to obtain which way information of the photon, without affecting its degrees of freedom like spacial-temporal shape and polarization. In the present paper, we shall consider the schemes of the arrangements based on the Mach-Zehnder interferometer and on the interferometer with two polarizing beam splitters. The legs of the interferometers are equipped with some devices, creating a Kerr medium to make the QND measurement of a single photon. The QND measurement occurs in the configuration other than the configuration of the interferometer, allowing to obtain which way information of the photon while preserving the work of the interferometer. 


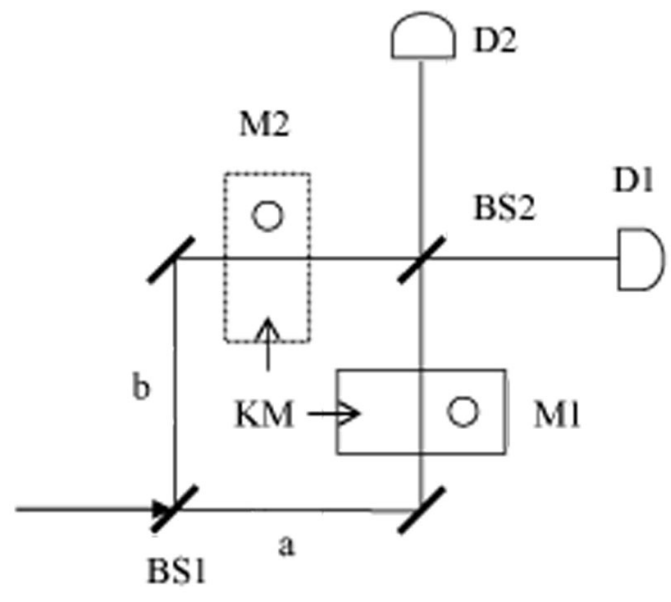

Figure 1: Scheme of the arrangement based on the Mach-Zehnder interferometer. BS: beam splitter, KM: Kerr medium, M: meter, D: detector.

\section{Schemes of the arrangements}

Consider the scheme of the arrangement based on the Mach-Zehnder interferometer equipped with some devices, creating a Kerr medium, one device on each leg, see Fig. 1. A cavity with an atom can be used as such a device [14]. In the standard Mach-Zehnder interferometer, a single photon is split into two components at the first 50/50 non-polarizing beam splitter. The components of the photon on legs $a$ and $b$ form the superposition state

$|\psi\rangle=\left(\left|\psi_{a}\right\rangle+\left|\psi_{b}\right\rangle\right) / \sqrt{2}$

When coupling at the second 50/50 non-polarizing beam splitter, the two components interfere. Having the phase between the components tuned, the photon goes out of the interferometer through the bright port of the second beam splitter, with the unity probability, and through the dark port, with the null probability. In the scheme in Fig. 1, each component of the photon, traveling the leg of the interferometer, interacts with a meter in a Kerr medium. Specifically, we shall take the atom in the cavity as a meter.

Interaction of a single photon with the atom in the cavity can be realized with the technique of the QND measurement, based on the strong-coupling regime of cavity quantum electrodynamics utilized in $[15,16,17]$. The technique allows QND detection of the photon. After the interaction with the meter atoms, the components of the photon are reflected off the cavities and directed to the second beam splitter. When coupling the components of the photon and the meter atoms, their states become entangled. The composite states of the components of the photon and the meter atoms on legs $a$ and $b$ are given respectively by

$\left|\Psi_{a}\right\rangle=\left(\left|0_{a}\right\rangle\left|g_{a}\right\rangle+\left|1_{a}\right\rangle\left|e_{a}\right\rangle\right) / \sqrt{2}$

$\left|\Psi_{b}\right\rangle=\left(\left|0_{b}\right\rangle\left|g_{b}\right\rangle+\left|1_{b}\right\rangle\left|e_{b}\right\rangle\right) / \sqrt{2}$

where $|0\rangle$ and $|1\rangle$ are the states of the photon in the photon-number basis, $|g\rangle$ and $|e\rangle$ are the ground and excited states of the meter atom respectively. The states of the photon and the meter atom are correlated. The meter atoms on legs $a$ and $b$ are subjected to the projective measurements in the basis of the ground and excited states. The projective measurements on the meter atoms give indirectly which way information about the photon. Thus, the atoms in the cavities act as which way detectors.

The state of the photon reflected off the cavity does not change. The spacial-temporal shape and polarization of the reflected photon remain the same. There is no transfer of the energy-momentum from the photon to the which way detector (atom in the cavity). Therefore, coupling the components of the photon and the meter atoms does not affect the work of the interferometer. The superposition of the photon eq. (1) is a one-particle state in which the components of the photon are defined in the space basis. The superpositions of the photon and atom eqs. $(2,3)$ are two-particle states in which the components of the photon are defined in the photon number basis. The projective measurement on the meter atom makes collapse of the two-particle state but does not affect the one-particle state of the photon. One can observe interference due to the superposition state eq. (1) at the second beam splitter of the interferometer. In the scheme, interference and which way information can be obtained in the different configurations, in accordance with the Bohr's complementarity principle.

To this end, the two-slit experiment with entangled photons showed both interference and which way information of the photon [21]. One of the entangled photons was taken to form interference in the two-slit configuration, and the twin photon served a detector to obtain which way information of the first photon. Like the experiment [21], the proposed scheme draws on the which way detector entangled to the photon. The photon reflected off the cavity acquires the $\pi$-phase shift, conditioned on the excited state of the meter atom. Addition of the $\pi$-phase shift to one of the components on leg $a$ or leg $b$ results in the interchange of the bright and dark ports of the second beam splitter. To obviate the interchange of the bright and dark ports one can utilize the phase shifters placed after the cavities, one on each leg. Upon having the projective measurements on the meter atoms on legs $a$ and $b$ made, instrument readings are expected to indicate one of the meter atoms in the ground state and the other in the excited state. Depending on the results obtained, one should switch on the phase shifter on the leg, corresponding to the excited meter atom, to do the $-\pi$-phase shift on the component of the photon thus erasing the phase information about the interaction of the component of the photon and the meter atom. Having the phase information erased, one can observe interference like in the standard Mach-Zehnder interferometer. 


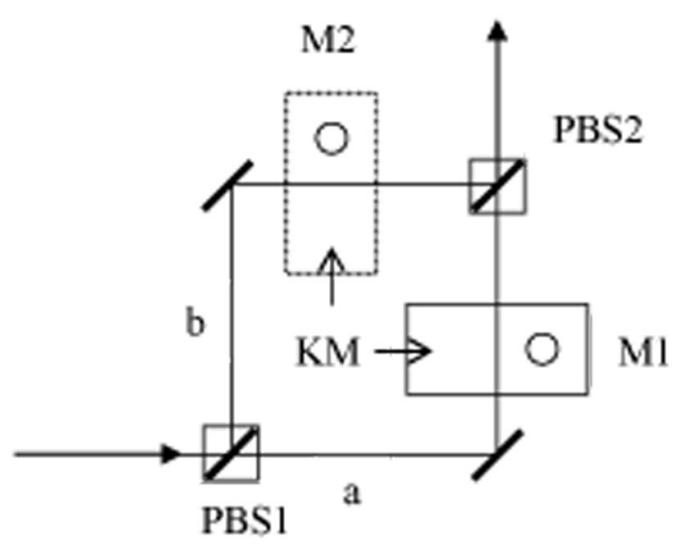

Figure 2: Scheme of the arrangement based on the interferometer with two polarizing beam splitters. PBS: polarizing beam splitter, KM: Kerr medium, M: meter.

Consider the scheme of the arrangement based on the interferometer with two polarizing beam splitters equipped with some devices, creating a Kerr medium, one device on each leg, see Fig. 2. The interferometer works as follows. Let a single photon enter the first polarizing beam splitter in the polarization state $|H\rangle+|V\rangle$ where $H$ and $V$ are horizontal and vertical polarizations respectively. After the first polarizing beam splitter, the components of the photon on legs $a$ and $b$ form the superposition state

$|\psi\rangle=\left(\left|H_{a}\right\rangle+\left|V_{b}\right\rangle\right) / \sqrt{2}$.

At the second polarizing beam splitter, the two components couple, restoring the initial state of the photon. In the scheme in Fig. 2, each component of the photon, traveling the leg of the interferometer, interacts with the meter in a Kerr medium. We shall take the atom in the cavity as a meter.

The composite states of the components of the photon and the meter atoms on legs $a$ and $b$ are given respectively by

$\left|\Psi_{a}\right\rangle=\left(\left|0_{H a}\right\rangle\left|g_{a}\right\rangle+\left|1_{H a}\right\rangle\left|e_{a}\right\rangle\right) / \sqrt{2}$

$\left|\Psi_{b}\right\rangle=\left(\left|0_{V b}\right\rangle\left|g_{b}\right\rangle+\left|1_{V b}\right\rangle\left|e_{b}\right\rangle\right) / \sqrt{2}$

The projective measurements on the meter atoms give indirectly which way and polarization information about the photon. The discussion of the scheme in Fig. 1 is applicable to the scheme in Fig. 2. One should use the phase shifters to compensate the $\pi$-phase shift of the photon reflected off the cavity. Then, the projective measurements on the meter atoms do not destroy the work of the interferometer, and the photon leaves the interferometer in the initial state.

The QND measurements in the arrangement in Fig. 2 give the polarization state of the photon within the interferometer $|H\rangle$ or $|V\rangle$ at random. Several passages of the photon through the arrangement in Fig. 2 collect statistics of the polarization state of the photon within the interferometer. From this, one can obtain the polarization state of the photon beyond the interferometer. Thus, the multiple passage of the photon through the arrangement in Fig. 2 allows to determine the polarization state of the photon. The scheme of the arrangement based on the interferometer with two polarizing beam splitters equipped with some devices, making the weak measurement, was considered in [10]. The arrangement can be used to perform an attack on the protocol BB84 [11, 12]. The attack on the protocol BB84 proposed in [11, 12] can be realized with the use of the arrangement in Fig. 2.

The proposed schemes draw on the entanglement as a resource for getting which way information of the photon in the QND measurement. In this case, the interferometer and the which way detector act in the different configurations, and their readings do not influence each other. Depending on the detector, the photon can be at the same time in the pure state (which way detector) and in the superposition state (interferometer). According to the interpretation of quantum mechanics [3, 4], a single photon follows a definite way (leg $a$ or leg $b$ ) at random while the superposition state eq. (1) describes the possibilities provided by the interferometer to the photon. It reflects the inability of the interferometer to reveal the way of the photon.

\section{Conclusion}

We have addressed the problem of which way information of the photon in the interferometer obtained by means of the QND measurement. We have considered the schemes of the arrangements based on the Mach-Zehnder interferometer and the interferometer with two polarizing beam splitters equipped with some devices to perform the QND measurement of a single photon. In the QND detection of the photon, there is no exchange of the energy-momentum between the photon and which way detectors. Therefore, the QND detection of the photon does not affect the work of the interferometer. In both the schemes, interference and which way information can be obtained in the different configurations, in accordance with the Bohr's complementarity principle. The arrangement based on the interferometer with two polarizing beam splitters allows to determine the polarization state of the photon without destroying it. The arrangement can be used to perform an attack on the protocol BB84. 


\section{References}

[1] N. Bohr, Atomic Physics and Human Knowledge, Science Editions, New York, 1961

[2] B.-G. Englert, Fringe visibility and which way information: An inequality, Phys. Rev. Lett. 77 (1996) $2154-2157$.

[3] D.L. Khokhlov, Interaction of the classical particle and quantum apparatus, Int. J. Gen. Syst. 44 (2015) 106-110.

[4] D.L. Khokhlov, Interference of a single photon in the Mach-Zehnder interferometer, Quan. Inf. Rev. 7 (2019) 7-10.

[5] Y. Aharonov, D.Z. Albert, L. Vaidman, How the result of a measurement of a component of the spin of a spin-1/2 particle can turn out to be 100, Phys. Rev. Lett. 60 (1988) 1351-1354

[6] Y. Aharonov, E. Cohen, A.C. Elitzur, Foundations and applications of weak quantum measurements, Phys. Rev. A 89 (2014) 052105.

[7] K.J. Resch, J.S. Lundeen, A.M. Steinberg, Experimental realization of the quantum box problem, Phys. Lett. A 324 (2004) $125-131$.

[8] A. Danan, D. Farfurnik, S. Bar-Ad, L. Vaidman, Asking photons where they have been, Phys. Rev. Lett. 111 (2013) 240402.

[9] D.L. Khokhlov, Modification of the two-slit experiment with a single photon, Optik, 126 (2015) 5301-5303.

[10] D.L. Khokhlov, Scheme of the weak measurement of the polarization state of the photon, Optik 127 (2016) 4089-4091.

[11] D.L. Khokhlov, Which way information in the non-perturbative post-measurement, Quantum Matter 4 (2015) $127-128$.

[12] D.L. Khokhlov, Scheme of the arrangement for attack on the protocol BB84, Optik 127 (2016) 7083-7087.

[13] P. Grangier, J.A. Levenson, J.-P. Poizat, Quantum non-demolition measurements in optics, Nature 396 (1998) $537-542$.

[14] S. Haroche, J.-M. Raimond, Cavity quantum electrodynamics, Scientific American 268 (1993) 54-60, 62.

[15] A. Reiserer, S. Ritter, G. Rempe, Nondestructive detection of an optical photon, Science 342 (2013) 1349-1351.

[16] S. Kono, K. Koshino, Y. Tabuchi, A. Noguchi, Y. Nakamura, Quantum non-demolition detection of an itinerant microwave photon, Nature Physics 14 (2018) 546-549.

[17] J.-C. Besse, S. Gasparinetti, M.C. Collodo et al., Single-shot quantum nondemolition detection of individual itinerant microwave photons, Phys. Rev. X 8 (2018) 021003.

[18] N. Matsuda, R. Shimizu, Y. Mitsumori, H. Kosaka, K. Edamatsu, Observation of optical-fibre Kerr nonlinearity at the single-photon level, Nature Photonics 3 (2009) 95-98

[19] G. Kirchmair, B. Vlastakis, Z. Leghtas et al., Observation of quantum state collapse and revival due to the single-photon Kerr effect, Nature 495 (2013) 205-209.

[20] J. Sinclair, D. Angulo, N. Lupu-Gladstein, K. Bonsma-Fisher, A.M. Steinberg, Observation of a large, resonant, cross-Kerr nonlinearity in a cold Rydberg gas, Phys. Rev. Res. 1 (2019) 033193.

[21] R. Menzel, D. Puhlmann, A. Heuer, W.P. Schleich, Wave-particle dualism and complementarity unraveled by a different mode, Proc. Natl. Acad. Sci. USA 109 (2012) 9314-9319. 